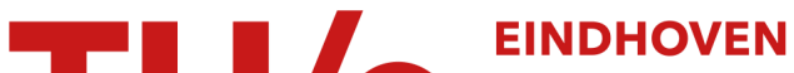 \\ UNIVERSITY OF \\ TECHNOLOGY
}

\section{Conjoint choice models of joint participation and activity choice}

Citation for published version (APA):

Dellaert, B., Borgers, A., \& Timmermans, H. (1996). Conjoint choice models of joint participation and activity choice. International Journal of Research in Marketing, 13(3), 251-264. https://doi.org/10.1016/01678116(96)00007-9, https://doi.org/10.1016/0167-8116(96)00007-9

DOI:

10.1016/0167-8116(96)00007-9

10.1016/0167-8116(96)00007-9

Document status and date:

Published: 01/07/1996

\section{Document Version:}

Publisher's PDF, also known as Version of Record (includes final page, issue and volume numbers)

\section{Please check the document version of this publication:}

- A submitted manuscript is the version of the article upon submission and before peer-review. There can be important differences between the submitted version and the official published version of record. People interested in the research are advised to contact the author for the final version of the publication, or visit the $\mathrm{DOI}$ to the publisher's website.

- The final author version and the galley proof are versions of the publication after peer review.

- The final published version features the final layout of the paper including the volume, issue and page numbers.

Link to publication

\section{General rights}

Copyright and moral rights for the publications made accessible in the public portal are retained by the authors and/or other copyright owners and it is a condition of accessing publications that users recognise and abide by the legal requirements associated with these rights.

- Users may download and print one copy of any publication from the public portal for the purpose of private study or research.

- You may not further distribute the material or use it for any profit-making activity or commercial gain

- You may freely distribute the URL identifying the publication in the public portal.

If the publication is distributed under the terms of Article $25 \mathrm{fa}$ of the Dutch Copyright Act, indicated by the "Taverne" license above, please follow below link for the End User Agreement:

www.tue.nl/taverne

Take down policy

If you believe that this document breaches copyright please contact us at:

openaccess@tue.nl

providing details and we will investigate your claim. 


\title{
Conjoint choice models of joint participation and activity choice
}

\author{
Benedict Dellaert ${ }^{\mathrm{a}, *}$, Aloys Borgers ${ }^{\mathrm{b}}$, Harry Timmermans ${ }^{\mathrm{b}, \mathrm{c}}$ \\ a Department of Marketing, The University of Sydney, Sydney, Australia \\ ${ }^{b}$ Urban Planning Group, Eindhoven University of Technology, Eindhoven, The Netherlands \\ ${ }^{c}$ Carthy Foundation Chair, Department of Marketing and Economic Analysis, University of Albertu, Faculty of Business Building, \\ Edmonton, Canada
}

Received 15 September 1994; accepted 27 February 1996

\begin{abstract}
Traditional conjoint choice models and experiments do not explicitly distinguish between modeling choices of whether or not to participate in a certain class of activities and choices of specific alternatives within that particular activity class. Experimental designs typically involve choices between several specific alternatives and a 'none' alternative, assuming that IIA holds between the utilities of specific alternatives and the 'none' alternative. Previous research has shown however that this assumption may be questionable (e.g. Bucklin and Lattin, 1991, Chintagunta, 1993). In this paper we present a framework to compare three possible conjoint choice modeling structures of joint participation and activity choice. These structures were tested in a case study on Dutch tourists' choices of outdoor flower exhibitions. It was found that contrary to commonly made assumptions in conjoint choice analysis an overall simple multinomial logit model was inadequate to model joint participation and activity choices. It was also found that after rescaling parameters estimated for participation choice were not significantly different from those estimated for activity choice and could be structured in an overall nested logit model.
\end{abstract}

Keywords: Choice-based conjoint analysis; Nested logit models; Participation choice

\section{Introduction}

In many areas of services marketing, models that adequately describe consumer activity choices can offer a valuable contribution in developing more effective marketing strategies. With this objective in mind, conjoint choice models have received substantial attention in marketing research over the past decade and promising results have been obtained in many different services marketing applications. Reviews of such applications have appeared in special issues of journals such as the Journal of Transport Economics and Policy (Bates, 1988), Leisure Sciences (Louviere and Timmermans, 1990) and the Journal of Business Research (Louviere, 1992).

\footnotetext{
" Corresponding author. E-mail: benedictd@bullwinkle.econ.su.oz.au; tel.: +61 2 351 4283; fax +61 23515632 . An earlier version of this paper was presented at the 1994 TIMS Marketing Science Conference, Tucson, AZ.
} 
In these applications, the choice of participating in a given activity class has typically been modeled identically to the choice of specific activities within activity classes. Usually a 'none' option is included to model non-participation as one of the alternatives in the designed choice sets. This is a base alternative that is present in each choice set and that offers respondents the possibility to indicate that they would prefer to choose none of the alternatives presented in the choice set. In modeling the consumer choice process it is implicitly assumed that the statistical properties of the 'none' alternative are identical to those of the specific activity alternatives and the observed proportion of 'none' choices is generally used to predict market penetration of an activity class similarly to how the observed proportions of choices of each activity are used to predict market shares. Moreover, as the simple multinomial logit model is commonly applied to describe the choice probabilities of both the 'none' alternative and the specific alternatives, it is assumed that independently and identically distributed (IID) Gumbel disturbances exist on the utility of the 'none' alternative and the specific activities.

Recently however, a number of studies applying econometric choice models to brand choice showed that the validity of these assumptions may be questioned. Bucklin and Lattin (1991), Chiang (1991) and Chintagunta (1993) for example tested model structures in which choices over time of whether or not to buy goods in a certain product category were modeled in combination with the choice of what to buy within that particular product category. They consistently found that parameters estimated on choices within product categories should be corrected when predicting choices of whether or not to buy in a certain product category and vice versa.

Although it could be argued that these studies applied econometric choice models rather than conjoint choice models the results they present do justify a strong suspicion against the validity of the commonly used assumptions underlying conjoint choice experiments that in a statistical sense the 'none' alternative can be treated equivalently to the specific activity alternatives. To the best of our knowledge, this issue has not been studied in a conjoint choice modelling context however and no specific designs have been developed to support tests and estimations of the proposed econometric models.

The purpose of this paper is therefore to present an extension of the traditional conjoint choice modeling approach that allows one to conduct critical tests of three basic model structures of service category participation choice combined with choices of specific service activities. More specifically the following models will be discussed, tested and compared: (i) a traditional simple multinomial logit model including a 'none' alternative and specific activities (e.g., Haider and Ewing, 1990; Louviere and Woodworth, 1983; Oppewal et al., 1994), (ii) a nested logit model in which activity choice is modeled conditionally on participation choice (Bucklin and Lattin, 1991; Chiang, 1991), and (iii) separate logit models of activity and participation choice (Gupta, 1988).

In Section 2 we will discuss the theoretical background of these three model structures and the experimental design strategy and estimation procedures that we propose to test the assumptions underlying the modeling structures. In Section 3 the results of an empirical test of the model will be presented and discussed. The paper closes with conclusions and a discussion of the proposed approach.

\section{Model formulation, experimental design and estimation}

In conjoint choice models, it is assumed that consumers choose to participate in those activities that represent the highest utility to them. It is furthermore assumed that the consumer choice process can be modelled as an evaluation process that integrates bundles of attributes describing goods and services (e.g., Louviere, 1988, 1994; Green and Srinivasan, 1990).

In our analysis we focus on the consumer choice of participating in a single category of service activities $a$, and the choice of specific activities $j$ within that category. Activities outside of class $a$ are considered as a composite alternative $\neg a$. The evaluation of this alternative is assumed to be independent and identical throughout the choice process. 
It is assumed that consumers base their choices on an evaluation of activity attributes, the utility of which consists of a structural part $V$, constant over time and common to all consumers, and a stochastic part $\epsilon$ that captures disturbances in utility due to taste variations between consumers and measurement errors. Different model structures can be generated to describe this process, depending upon the assumptions one is willing to make with regard to the structural and stochastic parts of the utility in consumers' participation and activity choices.

The most commonly applied approach in conjoint choice modeling is a model structure in which it is assumed that: (i) The value of the structural components in the utility functions are identical for consumers' activity choices and participation choices, (ii) The stochastic components of all activity alternatives as well as that of the non-participation alternative follow identically and independently distributed Gumbel distributions.

This is the simple multinomial logit (MNL) model and was applied in conjoint choice modeling for example by Haider and Ewing (1990), Louviere and Woodworth (1983) and Oppewal et al. (1994).

A second approach to model specific alternative choices in combination with category choices is the nested logit model (e.g., Bucklin and Lattin, 1991; Chiang, 1991). This approach is often suggested in econometric consumer choice modeling but has few applications in conjoint choice modeling. In the nested logit model it is assumed that: (i) The values of the structural components in the utility functions are identical for consumers' activity choices and participation choices. In contrast to the simple MNL model, the disturbances are allowed to vary between the choice types. It is therefore assumed that: (ii) The utilities of specific product or service alternatives follow IID Gumbel distributions, and can be regarded to be independently distributed elements within the disturbances on the utilities of categories, which are themselves also identically Gumbel distributed but with a larger variance.

As a third approach, specific product or services choices and category choices can be modelled as essentially different choice processes. In this case parameters are estimated for separate models. This implies that it is assumed that both (i) The structural components, and (ii) The stochastic components of consumers' utility functions can differ between participation and activity choices. Gupta (1988) for example, used this approach in a study of purchase incidence and brand choice. Table 1 summarizes the assumptions that are made in the three model structures with regard to the structural and stochastic components in consumers' utility functions.

In the next sections we will formally describe the three modeling approaches, and show how they are nested within each other in terms of model complexity. Firstly, we will formally describe the model structures. Secondly, we will discuss an experimental design strategy that supports estimation of the model structures and we will discuss more in depth the formal relationship that exists in logit type models between the structural and stochastic components of the utility function. It will be shown how this relationship has strongly impacted our proposed experimental design strategy and estimation approach. Thirdly, we will discuss an approach to estimating and comparing the proposed models.

\subsection{A simple multinomial logit model of participation and activity choice}

Let $J_{a}$ be the set of activities $j$ of activity class $a$. Let $U_{j}$ be the overall utility of alternative $j, V_{j}$ the structural utility of alternative $j$, and $\epsilon_{j}$ be the disturbance on the utility of alternative $j$. Let $U_{\neg a}$ be the overall

Table 1

Assumptions in proposed model structures of participation and activity choice

\begin{tabular}{lll}
\hline $\begin{array}{l}\text { Model structure for participation } \\
\text { and activity choice }\end{array}$ & $\begin{array}{l}\text { Identical structural utilities } V \\
\text { in participation and activity choice }\end{array}$ & $\begin{array}{l}\text { Identical stochastic components } \epsilon \\
\text { in participation and activity choice }\end{array}$ \\
\hline $\begin{array}{l}\text { Traditional simple multinomial } \\
\text { logit model }\end{array}$ & yes & yes \\
$\begin{array}{l}\text { Nested logit model } \\
\text { Separate logit models }\end{array}$ & yes & no \\
\hline
\end{tabular}


utility of not participating in activity class $a, V_{\neg a}$ the structural utility of not participating in activity class $a$, and $\epsilon_{\neg a}$ the disturbance on the utility of not participating in activity class $a$. Assume that all disturbances $\epsilon_{j}$ and $\epsilon_{\neg a}$ are independently and identically distributed (IID) according to a Gumbel distribution with mode 0 and scale parameter $\mu$. Assuming that consumers are maximizing their utility, the probability that alternative $j$ from the set of activities $J_{a}$ will be selected can be expressed as

$$
\begin{aligned}
P(j) & =P\left(U_{j} \leq U_{j^{\prime}} \wedge U_{j} \geq U_{\neg a} ; \forall j^{\prime} \in J_{a} ; j^{\prime} \neq j\right) \\
& =\frac{\exp \left(\mu V_{j}\right)}{\exp \left(\mu V_{\neg a}\right)+\Sigma_{j^{\prime} \in J_{a}} \exp \left(\mu V_{j^{\prime}}\right)}
\end{aligned}
$$

where $V_{j}=\boldsymbol{\beta} j^{\prime} \boldsymbol{x}_{j} ; \boldsymbol{\beta}_{j}$ is the parameter vector of the attributes describing alternative $j$, and, $\boldsymbol{x}_{j}$ is the vector of attributes of alternative $j$.

The probability of nol participating in an activity of class $a$ is

$$
\begin{aligned}
P(\neg a) & =P\left(U_{\neg a} \geq U_{j} ; \forall j \in J_{a}\right) \\
& =\frac{\exp \left(\mu V_{\neg a}\right)}{\exp \left(\mu V_{\neg a}\right)+\sum_{j^{\prime} \in J_{a}} \exp \left(\mu V_{j^{\prime}}\right)}
\end{aligned}
$$

where $V_{\neg a}=\boldsymbol{\beta}_{\neg a}^{\prime} x_{\neg a} ; \boldsymbol{\beta}_{\neg a}$ is the parameter associated with not participating in an activity of class $a$, and $\boldsymbol{x}_{\neg \mathrm{a}}$ is a dummy variable for not participating in an activity of class $a$. It can be easily seen that in this model structure $P(\neg a)+\sum_{j \in J_{a}} P(j)=1$.

\subsection{A nested multinomial logit model of participation and activity choice}

Let all elements be defined as before. Let $V_{j}=V_{\text {gen }}+V_{j}, \epsilon_{j}=\epsilon_{\text {gen }}+\epsilon_{j}$, and $U_{j} \cdot=V_{j} \cdot+\epsilon_{j}$, where $V_{\text {gen }}$ is the general component of the structural utility that is common to all activities in class $a$, but not to activities outside of $a, V_{j}$ is the structural utility specific to activity $j, \epsilon_{\text {gen }}$ and $\epsilon_{j}$. are disturbances on these utilities, and $U_{j}$. is the overall utility specific to activity $j$. Assume that all disturbances $\epsilon_{j}$. follow IID Gumbel distributions with mode 0 and scale parameter $\mu^{\text {lo }}$, that all $\epsilon_{j}$ are independent of $\epsilon_{\neg a}$ and that all $\epsilon_{j}$ $\left(=\epsilon_{\text {gen }}+\epsilon_{j} \cdot\right)$ and $\epsilon_{\neg a}$ are identically Gumbel distributed, with mode 0 and scale parameter $\mu^{\text {hi }}$. Then, the probability of choosing activity $j$, conditional on participating in an activity of class $a$ can be expressed as

$$
\begin{aligned}
P(j \mid a) & =P\left(U_{j^{*}} \geq U_{j^{\prime}} ; \forall j^{\prime} \in J_{a} ; j^{\prime} \neq j\right) \\
& =\frac{\exp \left(\mu^{\mathrm{lo}} V_{j^{*}}\right)}{\sum_{j^{\prime} \in J_{a}} \exp \left(\mu^{\mathrm{lo}} V_{j^{\prime}}\right)}
\end{aligned}
$$

where $V_{j},=\boldsymbol{\beta}_{j}^{\prime} \cdot \boldsymbol{x}_{j} ; \boldsymbol{\beta}_{j}$. is the vector of parameter values for attributes specific to alternative $j$, and $\boldsymbol{x}_{j} \cdot$ is the vector of attributes specific to alternative $j$.

If all disturbances $\epsilon_{j}$. follow IID Gumbel distributions with parameters $\left(0, \mu^{10}\right)$, all $U_{j}$. follow IID Gumbel distributions with parameters $\left(V_{j}, \mu^{10}\right)$. This implies that $\max _{j \in J_{a}}\left(U_{j}\right)$ is Gumbel distributed with parameters $\left(\left(1 / \mu^{\text {lo }}\right) \ln \sum_{j \in J_{a}}\left(\exp \left(\mu^{\text {lo }} V_{j}\right)\right), \mu^{\text {lo }}\right)$ (Johnson and Kotz, 1970). 
Since the probability of not participating in activity class $a$ is equal to the probability that the utility of not participating is higher than the utility of the most attractive alternative $j$ in class $a$, the probability of not participating can be expressed as

$$
\begin{aligned}
P(\neg a) & =P\left(U_{\neg a} \geq \max _{j \in J_{a}}\left(U_{j}\right)\right) \\
& =\frac{\exp \left(\mu^{\mathrm{hi}} V_{\neg a}\right)}{\exp \left(\mu^{\mathrm{hi}} V_{\neg a}\right)+\exp \left(\mu^{\mathrm{hi}}\left(V_{\mathrm{gen}}+\left(1 / \mu^{\mathrm{lo}}\right) \ln \sum_{j \in J_{a}} \exp \left(\mu^{\mathrm{lo}} V_{j^{*}}\right)\right)\right)}
\end{aligned}
$$

where $V_{\text {gen }}=\boldsymbol{\beta}_{\text {gen }}^{\prime} \boldsymbol{x}_{\text {gen }} ; \boldsymbol{\beta}_{\text {gen }}$ is parameter vector of attributes general to all alternatives $j \in J_{a}, \boldsymbol{x}_{\text {gen }}$ is the vector of attributes general to alternatives $j \in J_{a}$, and all other elements are defined as above. In this model structure the probability of participating in activity class $a, P(a)$, equals $1-P(\neg a)$.

The nested logit model reduces to the simple multinomial logit model if $\mu^{\text {hi }}$ equals $\mu^{\text {lo }}$, which can be seen as follows:

$$
\begin{aligned}
& \exp \left(\mu^{\mathrm{hi}}\left(V_{\mathrm{gen}}+\left(1 / \mu^{\mathrm{lo}}\right) \ln \sum_{j \in J_{a}} \exp \left(\mu^{\mathrm{lo}} V_{j^{*}}\right)\right)\right), \quad \mu^{\mathrm{hi}}=\mu^{\mathrm{lo}}=\mu \\
& =\exp \left(\mu V_{\mathrm{gen}}\right) \cdot \sum_{j \in J_{a}} \exp \left(\mu V_{j^{*}}\right) \\
& =\sum_{j \in J_{a}} \exp \left(\mu\left(V_{\mathrm{gen}}+V_{j^{*}}\right)\right), \quad V_{j}=V_{\mathrm{gen}}+V_{j} \cdot \\
& =\sum_{j \in J_{a}} \exp \left(\mu\left(V_{j}\right)\right)
\end{aligned}
$$

where all elements are defined as before. Thus the simple multinomial logit model participation and activity choice as a model is a simplification of the nested logit model.

\subsection{Separate logit models for participation and activity choice}

In this model structure separate models are proposed for the activity and the participation choice. Let $U_{j a}$ be the overall utility of alternative $j$ in the activity choice, $V_{j u}$ the structural utility of alternative $j$ in the activity choice, and $\epsilon_{j a}$ be the disturbance on the utility of alternative $j$ in the activity choice. Assume that all disturbances $\epsilon_{j a}$ follow IID Gumbel distributions with mode 0 and scale parameter $\mu^{a}$. The probability that alternative $j$ will be selected in the activity choice process can then be expressed as

$$
\begin{aligned}
P(j) & =P\left(U_{j a} \geq U_{j^{\prime} a} ; \forall j^{\prime} \epsilon J_{a} ; j^{\prime} \neq j\right) \\
& =\frac{\exp \left(\mu^{\mathrm{a}} V_{j a}\right)}{\sum_{j^{\prime} \in J_{a}} \exp \left(\mu^{\mathrm{a}} V_{j^{\prime} a}\right)}
\end{aligned}
$$

where $V_{j a}=\boldsymbol{\beta}_{\mathrm{ja}}^{\prime} \boldsymbol{x}_{\mathrm{ja}} ; \boldsymbol{\beta}_{\mathrm{ja}}$ is the parameter vector of attributes of alternative $j$ in the activity choice, and $\boldsymbol{x}_{j a}$ is the vector of attributes of alternative $j$ in the activity choice.

Let $U_{j p}$ be the overall utility of alternative $j$ in the participation choice, $V_{j p}$ the structural utility of alternative $j$ in the participation choice and $\epsilon_{j p}$ be the disturbance on the utility of alternative $j$ in the participation choice. Let $V_{j p}=V_{\text {gen }}+V_{j p^{*}}, \epsilon_{j p}=\epsilon_{\text {gen }}+\epsilon_{j p^{*}}$ and $U_{j p^{*}}=V_{j p^{*}}+\epsilon_{j p^{*}}$, where $V_{\text {gen }}$ is the structural utility in the participation choice common to all activities in class $a$, but not to activities outside of $a, V_{j p}$ * is the structural utility in the participation choice specific to activity $j, \epsilon_{\mathrm{gen}}$ and $\epsilon_{j p^{*}}$ are the disturbances on 
these utilities, and $U_{j p}$. is the overall utility specific to activity $j$ in the participation choice. Let all other elements be defined as before. If we then assume that

(i) all disturbances $\epsilon_{j p}$. follow IID Gumbel distributions with mode 0 and scale parameter $\mu^{\text {lo }}$,

(ii) all $\epsilon_{j p}$ are independent of $\epsilon_{\neg a}$, and

(iii) all $\epsilon_{j p}$ and $\epsilon_{\neg a}$ are identically Gumbel distributed, with mode 0 and scale parameter $\mu^{\text {hi }}$,the probability of not participating in an activity of class $a$, equals

$$
\begin{aligned}
P(\neg a) & =P\left(U_{\neg a} \geq \max _{j \in J a}\left(U_{j p}\right)\right) \\
& =\frac{\exp \left(\mu^{\mathrm{hi}} V_{\neg a}\right)}{\exp \left(\mu^{\mathrm{hi}} V_{\neg a}\right)+\exp \left(\mu^{\mathrm{hi}}\left(V_{\mathrm{gen}}+\left(1 / \mu^{\mathrm{lo}}\right) \ln \sum_{j \in J_{a}} \exp \left(\mu^{\mathrm{lo}} V_{j p^{*}}\right)\right)\right)}
\end{aligned}
$$

where $V_{\text {gen }}=\boldsymbol{\beta}_{\text {gen }}^{\prime} \boldsymbol{x}_{\text {gen }} ; \boldsymbol{\beta}_{\text {gen }}$ is the parameter vector in the participation choice of attributes general to alternatives $j \in J_{a}, \boldsymbol{x}_{\text {gen }}$ is the vector of attributes in the participation choice general to alternatives $j \in J_{a}$, $V_{j p}=\boldsymbol{\beta}_{\mathrm{jp}}^{\prime} \boldsymbol{x}_{\mathrm{jp}}, \boldsymbol{\beta}_{j p}{ }^{*}$ is the parameter vector in the participation choice for attributes specific to alternative $j, \boldsymbol{x}_{j p}$. is the vector of attributes in the participation choice specific to alternative $j$, and all other elements are defined as above.

As before, the nested logit structure for participation choice reduces to a simple multinomial logit model if $\mu^{\mathrm{hi}}$ equals $\mu^{\mathrm{lo}}$, and $P(a)=1-P(\neg a)$. Also the separate models reduce to one overall nested or simple multinomial logit model if $U_{j a}$ equals $U_{j p}$ up to a constant $C$. This is the case if the parameter vector $\boldsymbol{\beta}_{j a}$ of the attributes of alternative $j$ in the activity choice equals the parameter vector $\boldsymbol{\beta}_{j p}$. of the attributes specific to alternative $j$ in the participation choice.

\subsection{Experimental design}

Conjoint choice experiments reported in the literature typically depend upon the simple multinomial logit model (e.g., Bates, 1988; Louviere and Woodworth, 1983; Louviere, 1988; Timmermans, 1984). A necessary and sufficient condition to estimate this model efficiently, is that the experimental design used to create the choice alternatives is orthogonal. This guarantees that the attributes within the choice alternatives vary independently. A commonly applied design strategy is therefore to create an orthogonal fractional factorial design and then place the profiles into choice sets. Typically, a base alternative, coded zero, is added to each choice set to obtain orthogonality not only between the attribute levels themselves, but also between their relative differences. All estimates are then made in relation to the base alternative.

Variable or constant choice set designs may be used to create the choice sets. In case of variable choice sets, $2^{N}$ designs, where $N$ is the total number of profiles, are typically applied to vary the presence or absence of the profiles in the choice sets, and the two levels indicate that an alternative can be either present or absent in the choice set. Thus, choice sets of varying size and composition are created.

In constant choice set designs, various approaches may be adopted. Two commonly applied techniques are: (i) Each alternative $i$ in the choice set of fixed size $s$ is described in terms of $j$ attributes with levels $l_{j i}$. The attributes are placed in choice sets of size $s$ according to a fractional factorial design in which all levels are varied independently. For example, if one wishes to conduct an experiment with two alternatives with three and four attributes, all with three levels, a $3^{7}$ fractional factorial design is created to construct choice sets in which attributes vary independently both within and between alternatives. (ii) Alternative profiles from $k$ identical fractional factorial designs are randomly combined to create choice sets of size $k$, preventing identical alternatives from being placed in the same choice set. In principle, randomization of attribute comparisons renders the marginals of the alternatives independent of each other. Independence between the attributes describing the various alternatives can be tested by calculating the correlations between the columns of the combined design profiles. If the marginals are fully independent the correlations are zero (Louviere, 1988). 
Recently, it has been shown that the same design strategies can be used to test more complex, non-IIA choice models. This can be done by introducing the effects of attribute levels of other alternatives from the choice set into the utility function of the alternatives. These effects are generally referred to as cross effects and availability effects (Lazari and Anderson, 1994). If the design is orthogonal between attributes of different alternatives, these effects can be estimated independently of the effect of the alternatives' own attributes. For example, Anderson et al. (1992) used a fractional factorial design to create choice sets of travel mode alternatives and estimated the effect of the availability of each travel mode in the choice set on the preferences for other travel modes. Similarly, Timmermans et al. (1992) have demonstrated how fractional factorial designs that are orthogonal within and between alternatives can be used to estimate cross-effects between alternatives that depict the influence of attribute levels of one alternative on the preferences for other alternatives. Anderson and Wiley (1992) and Lazari and Anderson (1994) provide locally optimal designs for similar experiments, where alternatives are described by respectively their brand name or a single attribute. They present a catalog of designs up to a practical numbers of alternatives.

Although the above approaches extend the number and type of effects that can be estimated in multinomial logit models, they share the basic assumption with the simple multinomial logit model that disturbances in the utility function of the alternatives follow IID distributions. This is a necessary condition for factorial experimental designs to support efficient estimation of parameter values (Dey, 1985).

This assumption may however give rise to serious biases in the model estimations, if in reality disturbances are interdependent and/or heteroscedastic. The effect of these biases on estimates in econometric choice models has been extensively discussed in the literature (e.g., Horowitz, 1981; Swait and Louviere, 1993). The fact that incorrect assumption of IID disturbances may also reduce the efficiency of estimations based on experimental designs has however hardly been recognized in conjoint choice applications.

In understanding the effect of these biases, it is critical to realize that in the simple multinomial logit model the estimated parameter values $\beta$ are confounded with the scale parameter $\mu$ and that only the product $\mu \beta$ can be estimated. In the estimation process the scale parameter $\mu$ is therefore generally set to 1 and the parameters $\beta$ are estimated in relation to this value (Ben-Akiva and Lerman, 1985, p. 71). As a consequence, estimations made in choice situations with identical parameter values $\beta$, but with different underlying scale parameters will not lead to identical parameter estimates. The same applies to estimations made in choice situations with different disturbances, as, in logit models, there is a direct relationship between the variance of the disturbance and the scale parameter $\mu$, expressed as

$\operatorname{var}(\epsilon)=\pi^{2} / 6 \mu^{2}$

If for example, there exist two choice situations with disturbances $\epsilon_{1}$ and $\epsilon_{2}$ and scale values $\mu_{1}$ and $\mu_{2}$, the assumption of equally distributed disturbances $\epsilon_{T}$ may lead to biases in the estimated parameter values. Swait and Louviere (1993) show that the Maximum Likelihood Estimator (MLE) will necessarily overpredict for the choice situations with the larger variance (i.e., smaller $\mu$ ) and underpredict for the situations with the smaller variance (i.e., larger $\mu$ ), where the degree of over- and underprediction depends on the number of observations made in each choice situation.

If we now look at the proposed model structures for joint participation and activity choice, it can be seen that only the simple multinomial logit model complies with the assumption of IID disturbances. Both the overall nested logit model and the separate logit models have different disturbances for participation and activity choice. In the nested logit model they are $\epsilon_{j}$. for activity choice and $\epsilon_{j}$ for participation choice, with scale values $\mu^{\text {lo }}$ and $\mu^{\text {hi }}$. In the separate models, the disturbances are $\epsilon_{j a}$ for activity choice and $\epsilon_{j p}$ for participation choice, with scale values $\mu^{a}$ and $\mu^{\text {hi }}$. In both models, the disturbance on the participation choice is assumed to be larger than the disturbance on the activity choice. Therefore, if the underlying choice structure follows one of these two models rather than the simple multinomial logit model, estimations based on a traditional experimental design that assumes IID disturbances will be biased. Estimates of parameters for activity choice will be lower than their 
actual value and estimates for parameters of participation choice will be higher than their actual value. It is important to stress that these biases may occur, even if in the design all attribute levels are varied independently.

To prevent these biases, separate estimations of the parameters for respectively activity and participation choice should be supported by the design. It can be seen that in the proposed model structures the IID assumption does hold within the activity and participation choice models, both in the overall nested logit model and in the separate models, so that separate designs for each of the two choice situations are a sufficient condition to support efficient estimation of the model parameters $\beta$.

A second, equally important, reason why separate estimations should be supported, is that in the structure of separate logit models, the underlying parameters for activity and participation choice are expected to be different, even after scale correction. In the models, it is implied that the vector of parameter values for activity choices $\beta_{j a}$, need not be identical to the vector of parameter values $\beta_{j p}$. of the activity component in the participation choice.

The experimental design approach we propose therefore consists of two subdesigns. In the first subdesign, choice sets are created that represent choices within activity class. Any of the described design strategies may be used to create this subdesign. A base alternative activity that is part of the design is added to each choice set. Respondents are asked to choose from the presented activities the one that they favor most. This design supports efficient estimation of the parameters of activity choice. The second subdesign is different from the first in two respects: (i) the base alternative is changed to the option of not participating, and (ii) the choice sets are reduced to the base alternative plus only one other alternative. In this subdesign, respondents are asked to choose between either participating in the described activity or not. This design supports efficient estimation of the parameters for participation choice as the nested logit structure reduces to a simple multinomial logit structure if only one alternative is present within each nest. Fig. 1 presents the basic structure of the proposed experimental design approach for a case of $n$ alternatives clustered in $N$ choice sets, where the number of alternatives $k$ other than the base is 2 .

This design approach creates two fractional factorial designs that allow for efficient estimation of separate model parameters for activity and participation choice. It also allows for efficient estimation of the overall simple multinomial logit model across both subdesigns as orthogonality is maintained if the two fractional factorial designs are combined. The ratio of the scales in the nested logit model, which is $\mu^{\text {hi }}=\mu^{\text {lo }}$ both in the separate nested logit model for participation choice and in the overall nested logit model, can be estimated independently of the attribute levels, by comparing the parameter estimates in each of the subdesigns in one overall model. A scale parameter can then be estimated over the parameters of the first and second subdesign.

\subsection{Estimation}

The three model structures are nested within each other in terms of model complexity. This implies that observed reductions in model fit between the different model structures can be tested using the likelihood ratio test statistic (Theil, 1971) expressed as $2\left[L^{*}\left(\beta_{1}\right)-L^{*}\left(\beta_{2}\right)\right]$, where $L^{*}\left(\beta_{1}\right)$ and $L^{*}\left(\beta_{2}\right)$ are the adjusted log-likelihoods of the models under comparison, and which is asymptotically chi-square distributed.

First, separate models can be estimated for participation choice and activity choice. This requires estimation of parameters in separate simple multinomial logit models of activity and participation choice. This is supported in the design structure and the simple multinomial logit model can be estimated using standardly available commercial software. Secondly, one overall model is estimated over both choice tasks. Observations from both subdesigns are combined in a nested logit structure, in which activity choice is modeled conditional on participation choice. This is done by estimating one model across both subdesigns under the assumption of the overall nested logit model that the underlying parameter values over attributes are identical up to a scale correction in the participation and activity choice. This implies that the $\beta$ 's estimated on activity choice only, are assumed to be identical to the $\beta$ 's estimated on the activity related attributes in the participation choice, or in terms of the separate logit model structure, that $\beta_{j a}$ equals $\beta_{j p^{*}}$ and that $\mu^{a}$ equals $\mu^{\mathrm{lo}}$. A likelihood ratio test of 


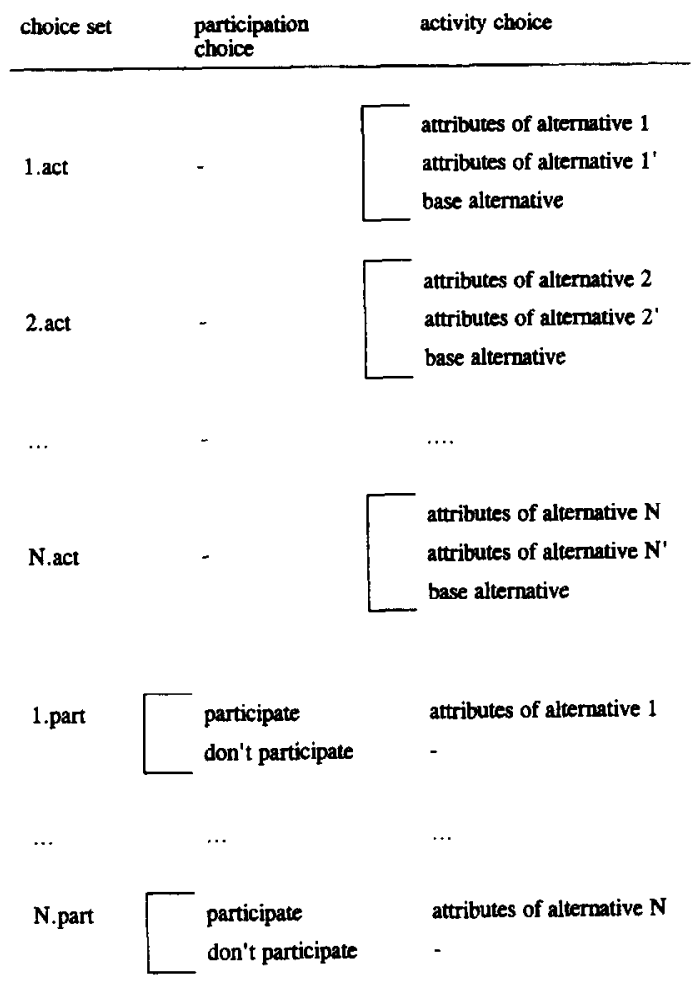

Fig. 1. Experimental design structure.

the overall nested logit model against the separate models can be conducted by comparing the fit of the overall nested logit model to the sum of the fits of the two separate models.

If the likelihood of the separate models is not significantly better than that of the overall nested logit model, a third test can be conducted in which the nested structure is tested against one overall simple multinomial logit model. This can be done by setting $\mu^{\text {hi }}$ equal to $\mu^{\mathrm{lo}}$ in the overall nested logit model and estimating an overall simple multinomial logit model. The likelihoods of the models can again be compared in a likelihood ratio test.

\section{An application to tourists' choices of outdoor flower exhibitions}

To test the viability of the proposed modeling framework, a case study on Dutch tourists' choices of large scale outdoor flower exhibitions was conducted. Parameters derived for choices made between competitive flower exhibitions only, were compared to parameters derived for choices of participating in one of the described exhibitions.

\subsection{Introduction}

In the Netherlands two types of large scale outdoor flower exhibitions are commonly held. The first type consists of annual exhibitions on permanent locations that represent popular day-trips. The most famous of its kind is the Keukenhof, located in Lisse, a small town near to Amsterdam. The second type of exhibitions is held less frequently at unique and varying locations. The largest in its kind is the Floriade, which is an official world-exhibition in the area of gardening. It is held once every ten years. Often, several Dutch towns compete 
as potential candidates for housing the Floriade, and it is generally used as a show case for municipal extension projects. One of the candidates for the 2002 Floriade was Eindhoven, a city in the southern part of the Netherlands. In the study, this city was used as location for the various alternatives that were presented to respondents.

\subsection{Method}

Respondents were presented with various descriptions of the Floriade. Three attributes were introduced to describe the alternatives: (i) entrance fee, (ii) presence of a special environmental issues exhibition and (iii) introduction of new public transportation to the exhibition. All three attributes represent features that have been used in the past as marketing tools in towns where the Floriade has been held, and are real points of consideration in the organization of the 2002 Floriade.

Entrance fee was varied over three levels ( $N L G$ 15, NLG 25 and NLG 35), and 'environmental exhibition' and 'new public transportation' were varied over two levels ( present, not present). Hypothetical day trips were formulated by varying attributes according to a $3.2^{2}$ full factorial design. The levels in the design were defined in orthogonal coding. For activity choices, choice sets were created per respondent by randomly combining alternatives drawn from two identical experimental designs that described profiles of different Floriade alternatives. Combinations of identical alternatives were not included in the choice sets and the Keukenhof with an entrance fee of NLG 15 was presented as a base alternative. For participation choices profiles were randomly drawn from the full factorial design and combined with the 'don't participate' alternative. This procedure guaranteed orthogonality of the attribute levels within alternatives. Respondents were asked to indicate which of the activities they were most likely to visit and whether they would or would not visit any of the exhibitions described in each set. The order of presentation of activity and participation choices was randomized over the respondents. This ensures that no systematic confounding takes place of possible task order effects and differences in the evaluation process between participation choices and activity choices.

Data for the study were collected in May 1994 through personal interviews. A convenience sample of 64 randomly drawn respondents from the Eindhoven region participated in the study. All were screened on having previously visited the Floriade or Keukenhof and all respondents were presented with all alternatives.

\subsection{Results}

First, separate models were estimated for activity and participation choice. Table 2 presents the parameters estimated in a simple multinomial logit model of activity choice. Choices were made between the various descriptions of the Floriade and the base alternative Keukenhof. Table 3 presents parameter values estimated for

Table 2

Parameters estimated for the separate simple multinomial logit model of activity choice (three alternatives) ${ }^{\text {a }}$

\begin{tabular}{lccc}
\hline Attribute & Parameter estimate & Standard ertor & $t$-value \\
\hline Floriade specific constant & 0.839 & 0.164 & 5.105 \\
Costs: & & & -12.465 \\
linear & -2.128 & 0.171 & 0.011 \\
quadratic & 0.007 & 0.069 & 6.018 \\
Environmental exhibition & 0.658 & 0.109 & 3.686 \\
New public transportation & 0.384 & 0.104 & \\
\hline
\end{tabular}

a $L^{*}(0):-419.6699 ; L^{*}(\hat{\beta}:-225.6622 ;$ McFadden's RhoSq: 0.4623. 
Table 3

Parameters estimated for the separate simple multinomial logit model of participation choice (two alternatives) ${ }^{a}$

\begin{tabular}{lccc}
\hline Attribute & Parameter estimate & Standard error & $t$-value \\
\hline Participation specific constant & 0.319 & 0.082 & 3.884 \\
Costs: & & & -10.955 \\
linear & -1.159 & 0.106 & 0.305 \\
quadratic & 0.017 & 0.055 & 2.089 \\
Environmental exhibition & 0.169 & 0.081 & 2.407 \\
New public transportation & 0.195 & 0.081 & \\
\hline
\end{tabular}

${ }^{\mathrm{a}} L^{*}(0):-532.3370 ; L^{*}(\hat{\beta}):-450.0965:$ McFadden's RhoSq: 0.1545 .

the simple multinomial logit model of participation choice in the single Floriade alternatives described in the choice situations.

In both choice tasks parameters had signs as expected. Choice probabilities decreased with higher costs, and increased with the introduction of an environmental exhibition and a new public transportation system. The positive Floriade specific constant in Table 2 indicates that respondents on average had a preference for visiting the Floriade over visiting the Keukenhof and the participation specific constant in Table 3 indicates that respondents on average also had a preference for visiting the Floriade over not participating.

The result of the overall estimation over both choice tasks is presented in Table 4 . It was found that the separate models of the two choice tasks did not perform significantly better than one overall rescaled model. In a likelihood ratio test the log-likelihood of the overall nested logit model was tested against the two separate multinomial logit models: $2\left(L^{*}(\right.$ separate $)-L^{*}($ nested overall $\left.)\right)=2(-675.7587--677.5676)=3.6178$, which is not significant at the 0.05 level for $v=3$. Therefore the overall nested logit model with activity choices nested under participation choice was accepted in favor of the separate logit models of activity and participation choice.

The estimated ratio between the scales $\left(\mu^{\mathrm{hi}} / \mu^{\mathrm{lo}}\right)$ of the separate activity and participation choice models was found to be 0.5220 which implies that the variance over the participation choices was on average $1 /\left(\begin{array}{ll}0.5220 & 2\end{array}\right)$ $=3.6703$ times that over the activity choice and that parameters estimated in the participation choice were on average 0.5220 times smaller than those estimated in the activity choices.

In a second likelihood ratio test the nested logit structure was then compared to the overall simple multinomial logit model: $2\left(L^{*}(\right.$ nested overall $)-L^{*}($ simple overall $\left.)\right)=2(-677.5676--692.6054)=$ 30.0757 , which is highly significant at the 0.05 level for $v=1$. Therefore the overall simple multinomial logit

Table 4

Combined parameter estimates overall nested logit model ${ }^{\text {a }}$

\begin{tabular}{lccc} 
Attribute & Parameter estimate & Standard error & $t$-value \\
\hline Floriade specific constant & 0.432 & 0.085 & 5.055 \\
Participation specific constant & 0.320 & 0.081 & 3.947 \\
Costs: & & & -16.615 \\
$\quad$ linear & -1.120 & 0.067 & 0.307 \\
quadratic & 0.009 & 0.030 & 6.365 \\
Environmental exhibition & 0.290 & 0.046 & 4.398 \\
New public transportation & 0.197 & 0.045 & \\
\hline
\end{tabular}

${ }^{a}$ Scale factor activity v.s. participation choice: $0.5220 . L^{*}(0):-952.0069 ; L^{*}(\hat{\beta}):-677.5676$; McFadden's RhoSq: 0.2883 . 
Table 5

Combined parameter estimates overall simple multinomial logit model a

\begin{tabular}{lccr}
\hline Attribute & Parameter estimate & Standard error & $t$-value \\
\hline Floriade specific constant & 0.863 & 0.150 & 5.738 \\
Participation specific constant & 0.366 & 0.087 & 4.199 \\
Costs: & -1.494 & 0.087 & -17.188 \\
$\quad$ linear & & & 0.237 \\
quadratic & 0.010 & 0.041 & 5.506 \\
Environmental exhibition & 0.345 & 0.063 & 4.167 \\
New public transportation & 0.258 & 0.062 & \\
\hline
\end{tabular}

${ }^{a} L^{*}:(0):-952.0069 ; L^{*}(\hat{\beta}):-692.6054 ;$ McFadden's RhoSq: 0.2725 .

model was rejected. For reasons of comparison the estimates of the traditional overall simple multinomial model are presented in Table 5.

\subsection{Validity test}

To test the validity of the parameter estimates and the design approach, a third subdesign was presented to the respondents. It was similar in plan to the proposed experimental design approach and represented a hybrid form of the earlier discussed subdesigns. Respondents were presented with two Floriade alternatives and a base of not participating, so that the choice task involved evaluation of activities and participation versus non-participation. The structure of the design used in the validity test is represented in Fig. 2.

Predictions made on the basis of the overall simple multinomial logit model, the nested logit model and separate logit models for participation and activity choice, were tested on choice data from the third subdesign. In making the predictions for the separate logit models $\beta$ parameters were set at the values previously estimated for the separate activity and participation choices and the scale parameter $\mu$ in the separate participation choice was set equal to that in the overall nested logit model. Observed choice frequencies were compared with predicted frequencies. The log-likelihoods for the model structures on the hold out data were calculated and are presented in Table 6. Table 7 shows the log-likelihood ratio test statistics of the different model structures. It

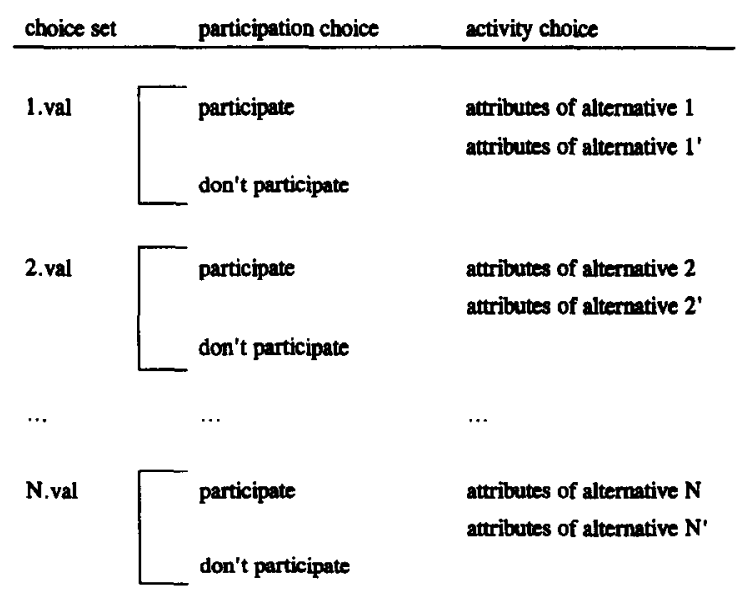

Fig. 2. Experimental design structure of validity test. 
Table 6

Log-likelihoods of the models on hold-out data

\begin{tabular}{ll}
\hline Model & Log-likelihood on hold-out data \\
\hline Null-model & -419.669 \\
Simple overall multinomial logit & -305.262 \\
Overall nested logit & -292.611 \\
Separate logit models & -291.526 \\
\hline
\end{tabular}

Table 7

Log-likelihood ratio test statistics of the models on hold-out data

\begin{tabular}{llll}
\hline Model & Null model & Simple-MNL & Nested-MNL \\
\hline Simple overall multinomial logit & 228.814 & - & - \\
Overall nested logit & 254.116 & 25.302 & - \\
Separate logit models & 256.286 & 27.472 & $2.170^{*}$ \\
\hline
\end{tabular}

* Not significant at the 0.05 reliability level.

can be seen that the overall nested logit model outperformed the overall simple multinomial logit model significantly and that its predictions were not significantly worse than those of the separate logit models.

\section{Conclusions and discussion}

This study compared three model structures to describe service category participation and specific activity choices: (i) a simple multinomial logit model including both a 'none' alternative and specific activities, (ii) a nested logit model with activity choice nested conditionally on participation choice, and (iii) a structure of separate logit models for participation and activity choices. A specific experimental design approach was proposed to support tests of the model structures in conjoint choice analysis applications.

The model structures were compared and tested in a case study using the proposed experimental approach. The results showed that the overall nested logit model offered the best explanation of the observed participation and activity choices. This finding was supported by the results of a validity test consisting of a task of combined activity and participation choices.

More specifically, the results showed that without scale corrections, models describing consumer choices within a given class of activities cannot be used adequately to predict participation choices and vice versa. It is therefore recommendable in conjoint analysis to estimate parameters for at least several identical attributes in both activity and participation choice situations, so that a scale correction can be estimated between the two choice types.

If our findings can be generalized, they imply that assumptions commonly made in conjoint choice models in which a 'none' or 'don't participate' base alternative is applied should be reconsidered, as they generally apply single overall simple multinomial logit models to model both activity and participation choices. More specifically, it can be expected that these have overestimated the effect of changes in activity attributes on participation choices and have underestimated the effect of these changes on choices of specific activities. This is due to the fact the estimated parameters are a weighted average of the differing underlying parameters for participation and activity choices.

In terms of marketing management the results imply that generally speaking considerably larger efforts are required to draw consumers into certain activity categories than to persuade them to switch between different activities within a category. Consequently, the introduction of new services within a certain service category 
will more strongly impact the competition level within that category than that relative to other categories. In the case study it was shown that this effect could adequately be explained by rescaling the parameters for category participation choices relative to the parameters for choices within a given category of activities. On the basis of this, marketing managers may want to reconsider their existing conjoint choice based models and try and correct for the likely overprediction in those models of the impact of marketing strategies on consumer participation choice and the underprediction of the impact of such strategies on consumers' choices of specific activities.

\section{References}

Anderson, D.A. and J.B. Wiley, 1992. Efficient choice set designs for estimating cross-effects models. Marketing Letters 3, $357-370$.

Anderson, D., A. Borgers, D. Ettema and H. Timmermans, 1992. Estimating availability effects in travel choice modeling: A stated choice approach. Transportation Research Record 1357, 51-65.

Bates, J.J., 1988. Econometric issues in stated preference analysis. Journal of Transport Economics and Policy 22, 59-71.

Ben-Akiva, M. and S. Lerman, 1985. Discrete choice analysis: Theory and application to travel demand. Cambridge, MA: MIT Press.

Bucklin, R.E. and J.M. Lattin, 1991. A two-state model of purchase incidence and brand choice. Marketing Science 10, 24-39.

Chiang, J., 1991. A simultaneous approach to the whether, what and how much to buy questions. Marketing Science 10, $297-315$.

Chintagunta, P.K., 1993. Investigating purchase incidence, brand chnice and purchase quantity decisions of households. Marketing Science $12,184-208$.

Dey, A., 1985. Orthogonal fractional factorial designs. New Delhi: Wiley Eastern Limited.

Green, P. and V. Srinivasan, 1990. Conjoint analysis in marketing: New developments with implications for research and practice. Journal of Marketing 54, 3-19.

Gupta, S., 1988. Impact of sales promotions on when, what, and how much to buy. Journal of Marketing Research 25, 341-355.

Johnson, N. and S. Kotz, 1970. Distributions in statistics: Continuous univariate distributions, Vol. 1. New York: John Wiley and Sons.

Haider, W. and G.O. Ewing, 1990. A model of tourist choices of hypothetical Carribean destinations. Leisure Sciences 12, 33-47.

Horowitz, J., 1981. Sampling, specification and data errors in probabilistic discrete choice models. In: D. Hensher and L. Johnson, eds., Applied discrete-choice modelling, 417-435. New York: Wiley.

Lazari, A. and D.A. Anderson, 1994. Designs of discrete choice set experiments for estimating both attribute and availability cross effects. Journal of Marketing Research 31, 375-383.

Louviere, J.J., 1988. Analyzing decision making: Metric conjoint analysis. Beverly Hills, CA: Sage Publications.

Louviere, J.J., 1992. Experimental choice analysis: Introduction and overview. Journal of Business Research 24, 89-95.

Louviere, J.J., 1994. Conjoint analysis. In: R.P. Bagozzi, Advanced methods in marketing research, 223-259. Cambridge: Blackwell Publishers.

Louviere, J.J. and H.J.P. Timmermans, 1990. Stated preference and choice models applied to recreation research: A review. Leisure Sciences $12,9-32$.

Louviere, J.J. and G. Woodworth, 1983. Design and analysis of simulated consumer choice of allocation experiments. Journal of Marketing Research 20, 350-367.

Oppewal, H., J.J. Louviere and H.J.P. Timmermans, 1994. Modeling hierarchical conjoint processes with integrated choice experiments. Journal of Marketing Research 31, 92-105.

Swait, J. and J.J. Louviere, 1993. The role of the scale parameter in the estimation and use of generalized extreme value models. Iournal of Marketing Research 30, 305-314.

Theil, H., 1971. Principles of econometrics. New York: Wiley.

Timmermans, H.J.P., 1984. Decompositional multiattributc preference models in spatial choice analysis: A review of some recent trends. Progress in Human Geography 8, 189-221.

Timmermans, H.J.P., A.W.J. Borgers and P.J.H.J. van der Waerden, 1992. Mother logit analysis of substitution effects in consumer shopping destination choice. Journal of Business Research 24, 177-189. 